\title{
Prenatal exposure to perfluoroalkyl and polyfluoroalkyl substances and childhood atopic dermatitis: a prospective birth cohort study
}

Qian Chen ${ }^{1}$, Rong Huang ${ }^{1}$, Li Hua ${ }^{2}$, Yifeng Guo ${ }^{3}$, Lisu Huang ${ }^{4,5}$, Yanjun Zhao ${ }^{6}$, Xia Wang $^{1}$ and Jun Zhang ${ }^{1 *}$

\begin{abstract}
Background: Perfluoroalkyl and polyfluoroalkyl substances (PFASs) have been reported to suppress immune function. However, previous studies on prenatal exposure to PFASs and allergic disorders in offspring provided inconsistent results. We aimed to examine the association between prenatal exposure to PFASs and childhood atopic dermatitis $(A D)$ in offspring up to 24 months of age.

Methods: A prospective birth cohort study involving 1056 pregnant women was conducted in two hospitals in Shanghai from 2012 to 2015. Prenatal information was collected by an interview with the women and from medical records. Fetal umbilical cord blood was collected at birth. Cord blood plasma PFASs were measured. Children were followed at 6, 12 and 24 months and information on the development of AD was recorded. AD was diagnosed by 2 dermatologists independently based on the questionnaires. Multiple logistic regression was used to compute odds ratio (OR) and corresponding 95\% confidence interval (Cl) for the association between AD and each PFASs, adjusting for potential confounders.
\end{abstract}

Results: A total of 687 children completed a 2-year follow-up visit and had PFASs measurement. AD was diagnosed in 173 (25.2\%) children during the first 24 months. In female children, a log-unit increase in perfluorooctanoic acid (PFOA) was associated with a 2.1-fold increase in AD risk (AOR 2.07, 95\% Cl 1.13-3.80) after adjusting for potential confounders. The corresponding risk was $2.22(1.07-4.58)$ for perfluorononanoic acid (PFNA). The highest PFOA quartile was significantly associated with $\operatorname{AD}(2.52,1.12-5.68)$ compared with the lowest quartile. The highest quartile of PFNA, perfluorodecanoic acid (PFDA) and perfluorohexane sulfonic acid (PFHxS) were associated with AD with AOR (95\% Cl) being 2.14 (0.97-4.74), 2.14 (1.00-4.57), and 2.30 (1.03-5.15), respectively. Additionally, the second quartile of perfluorododecanoic acid (PFDoA) was associated with a 3.2-fold increase in AD risk (3.24, 1.44-7.27). However, no significant associations were found in male children.

Conclusions: Prenatal exposure to PFOA, PFDA, PFDoA and PFHxS significantly increased the risk of childhood AD in female children during the first 24 months of life. In addition, the associations between AD with prenatal exposure to PFNA were close to statistical significance.

Keywords: Perfluoroalkyl and polyfluoroalkyl substances, Cord blood, Exposure, Atopic dermatitis, Childhood

\footnotetext{
* Correspondence: junjimzhang@sina.com; zhangjun@xinhuamed.com.cn

${ }^{1}$ Ministry of Education-Shanghai Key Laboratory of Children's Environmental Health, Xinhua Hospital, Shanghai Jiao Tong University School of Medicine, 1665 Kongjiang Road, Shanghai 200092, China

Full list of author information is available at the end of the article
} 


\section{Background}

Perfluoroalkyl and polyfluoroalkyl substances (PFASs) are synthetic compounds with strong fluorine-carbon covalent bonds. PFASs have strong thermal, biological, and chemical stability as well as hydrophobic and lipophobic properties, and have been widely used worldwide in industrial (lubricants, surfactants) and consumer products such as nonstick cookware, waxes, paints, cosmetics and as water and oil repellents for leather, paper, and textiles since 1950s [1]. Most of the PFASs members are resistant to degradation and bio-accumulative and are ubiquitous in environment such as air, water, sediment and sludge; as well as wildlife [2]. The half-lives in human bodies $(\mathrm{t} 1 / 2)$ of three common PFASs, perfluorooctanoic acid (PFOA), perfluorooctane sulfonate (PFOS) and perfluorohexane sulfonate (PFHxS), are 3.8, 5.4 and 8.5 years, respectively [3]. Although the production of PFOA and PFOS and related compounds has been phased out in some countries, the production of these chemicals or their precursors has actually increased in Asia, especially in China [4-6]. Environmental exposure to PFASs has been of increasing public health concern.

Extensive researches have associated exposure to PFASs with immunotoxicity, hepatotoxicity, developmental toxicity, reproductive toxicity, neurotoxicity, endocrine toxicity, liver tumor, impaired thyroid function, and mammary glands development [7-11]. Experimental studies suggested that PFOA and PFOS may cause immune suppression at doses of daily exposure in human and animals $[12,13]$. However, findings from epidemiologic studies were far from consistent $[14,15]$. Furthermore, these were cross-sectional or case-control studies, and evidence of PFASs causing allergic disorders is still limited, particularly from prospective studies.

Atopic dermatitis (AD) is a skin disease due to skin barrier dysfunction, resulting in eczematous and itchy lesions at the flexural folds and other typical distributions [16]. It is often caused by a combination of immune dysfunction and environment. Most AD occurs before 1 year of age, and it is generally considered as the first sign of the development of allergic diseases, including asthma, allergic rhinitis and conjunctivitis $[17,18]$. The prevalence of AD ranges from $2.4 \%$ to $37 \%$ in children and $6.8 \%-10.6 \%$ in adults worldwide $[19,20]$, and was $12.9 \%$ in children aged 1-7 years in China in a previous study [21].

Given the facts that fetuses are more vulnerable to PFASs and PFASs might impair immune function [22], we investigated the effects of prenatal exposure to PFASs on $\mathrm{AD}$ in children up to 24 months of age in a prospective cohort study in Shanghai, China.

\section{Methods}

\section{Study design}

A prospective birth cohort study was conducted in two large tertiary-level hospitals in Shanghai from 2012 to
2015. Women were recruited if they had a singleton pregnancy and planned to live in Shanghai for at least 2 years. A written consent was obtained. Trained nurses conducted face-to-face interviews and collected information on age, parental education levels and family income, parental history of atopic diseases (i.e. AD, allergic rhinitis, hives or asthma), alcohol use, smoking status, including secondhand smoke and active smoking during pregnancy. At birth, trained nurses measured newborn's anthropometric parameters and collected umbilical cord blood. Information was also collected from hospital records with patients' consent.

A total of 1056 baby-mother pairs were enrolled in the study. Two hundred forty-five children were excluded due to missing data on birthweight $(n=3)$ or loss to follow-up by 24 months old, resulting in 811 (76.8\%) for further data analyses.

Cases of AD were defined by the International Study of Asthma and Allergies in Childhood (ISAAC) questionnaire through three questions: "Has your child ever had an itchy rash which was coming and going for at least six months at any time?", "Has the itchy rash been coming and going over elbows, knees, face, wrists, or generalized (4 or more localizations)?", and "Has your child ever had atopic dermatitis diagnosed by a doctor?" [23]. Pictures of child AD were used to aid parental reporting. Two dermatologists diagnosed AD independently based on the questionnaires for all the children. Disagreements were resolved by consensus.

\section{Questionnaire survey}

At the 6-month follow-up, an online survey was conducted to collect information on duration of breastfeeding, consumption of fruits, vegetables, egg, wheat, soy bean, and shrimp before 6 months of age, living environment and post-natal environmental smoke exposure. AD related questions from ISAAC were also asked. At 12month and 24-month follow-up visits, face-to-face interviews were conducted to collect similar information as that in 6-month follow-up visit.

\section{Sample collection and preparation}

Blood samples were collected at delivery by double clamping the umbilical cord at the placental and infant ends. For plasma preparation, cord blood was collected in sterile EDTA tubes and centrifuged (10 min, $2400 \mathrm{~g})$. Plasma was separated and stored at $-80{ }^{\circ} \mathrm{C}$ until delivery to the research laboratory for PFASs analysis.

\section{Measurement of PFASs concentration}

A total of 10 PFASs were measured, including PFOA, PFOS, perfluorononanoic acid (PFNA), perfluorodecanoic acid (PFDA), perfluoroundecanoic acid (PFUA), PFHxS, perfluorooctane sulfonamide (PFOSA), perfluorododecanoic 
acid (PFDoA), perfluorobutane sulfonic acid (PFBS) and perfluoroheptanoic acid (PFHpA). The PFASs and internal standard solution $\left({ }^{13} \mathrm{C} 4\right.$-PFOS and ${ }^{13} \mathrm{C} 4$-PFOA) were purchased from Wellington Laboratories (Guelph, Ontario, Canada) and Sigma-Aldrich (St. Louis, MO, USA). Purities of all standards were $\geq 95 \%$.

Detailed methods of PFASs measurements have been described in our previous report [24]. Briefly, the frozen samples were firstly thawed at room temperature and then vortex mixed for $15 \mathrm{~s}$ to ensure homogeneity. A $100 \mu \mathrm{L}$ plasma sample contained in a polypropylene centrifuge tube was vortexed with $10 \mu \mathrm{L}$ of $50 \mathrm{ng} / \mathrm{mL}$ internal standard solution for 30s. Then $150 \mu \mathrm{L}$ of methanol and $150 \mu \mathrm{L}$ acetonitrile containing $1 \%$ formic acid were added to each sample before the second vortex. The mixture was sonicated for $10 \mathrm{~min}$ and then centrifuged at 12,000 rpm for $10 \mathrm{~min}$. The supernatant was collected $(\sim 100 \mu \mathrm{L})$ and then filtered through $0.22-\mu \mathrm{m}$ Nylon syringe filter into a $1.5 \mathrm{~mL}$ auto-sampler vial.

The calibration standard solution was prepared in $100 \mu \mathrm{L}$ of bovine serum and went through sample preparations under the same procedures. The final concentrations for all analytes were $0.5-100 \mathrm{ng} / \mathrm{mL}$ containing a fixed amount of internal standard $(25 \mathrm{ng} / \mathrm{mL})$ in bovine serum samples. The separation and detection were performed on high performance liquid chromatography with tandem mass spectrometry (HPLC-MS/MS, Agilent 1290-6490, Agilent Technologies Inc., U.S.A). A 2$\mu \mathrm{L}$ aliquot of the sample extract was injected into a ZORBAX Eclipse Plus C18 column $(2.1 \times 100 \mathrm{~mm}$, $1.8 \mu \mathrm{m}$; Agilent, USA) maintained at $35{ }^{\circ} \mathrm{C}$ and equipped with a C18 pre-column $(2.1 \times 5 \mathrm{~mm}, 1.8 \mu \mathrm{m})$. The flow rate was $0.3 \mathrm{~mL} / \mathrm{min}$. Mobile phases $\mathrm{A}$ and $\mathrm{B}$ were water contained $10 \mathrm{mM}$ ammonium acetate and methanol, respectively. The gradient elution started at $60 \%$ methanol and increased to $80 \%$ after $5 \mathrm{~min}$, then increased to $90 \%$ at $10 \mathrm{~min}$; it was held at $90 \%$ for $1 \mathrm{~min}$, and then returned back to $40 \%$ methanol at $12 \mathrm{~min}$. The instrument was operated in dynamic multiple reaction monitoring (MRM) with an electrospray ionization (ESI) negative mode.

Recovery rate ranged between $80-110 \%$. Intra- and inter-day coefficient of variations was below $10 \%$. The detection limits for PFASs ranged from 0.009 to $0.12 \mathrm{ng} / \mathrm{mL}$. For concentrations below the detection limits, a value of half the lower limit was assigned. PFOSA and PFHpA were detected in $<30 \%$ of the samples, and thus were not used for further analysis. PFOS, PFNA, PFHxS were detected in all samples, and the other 5 PFASs were detectable in $>90 \%$ of the samples.

\section{Statistical analysis}

Socio-demographic data and PFASs concentrations in cord blood plasma were compared between children with and without AD, by Wilcoxon Rank Sum test or Cochran-Mantel-Haenszel test, where appropriate. Medians (Q1, Q3) were calculated for PFASs. Because of the skewed distributions, a log transformation was performed for cord blood plasma PFASs concentrations for logistic regression.

The association of log-cord blood plasma PFASs concentrations and $\mathrm{AD}$ were analyzed by univariate and multivariate logistic regression models. PFASs concentrations were log transformed in the first model and categorized into quartiles with the lowest quartile as the reference level in the second model. Potential confounders were selected based on a directed acyclic graph, included infant sex, parity (nulliparous and parous), birth weight, gestational age at delivery, mode of delivery, maternal pre-pregnancy BMI, maternal age, maternal education, maternal ethnicity, paternal age, paternal education, parental history of allergic disorders, paternal smoking during pregnancy, family income and breasting during the first 6 months (Additional file 1: Figure S1). As only few women drank (1.98\%) and smoked $(0.5 \%)$, maternal drinking and smoking were not included in the fully adjusted model. All the analyses were performed using the SAS software, version 9.4 (SAS Institute, Inc., Cary, NC, USA).

\section{Results}

A total of 687 children completed a 2-year follow-up visit and had PFASs measurement. During the first 24 months, 173 children (25.2\%) developed AD. There were no significant differences between those followed up and the total population with regard to basic characteristics (shown in Additional file 2: Table S1). Table 1 shows that the mean maternal and paternal ages were 29 and 31 years, respectively. The majority of pregnant women had normal weight $\left(18.5 \leq \mathrm{BMI}<25 \mathrm{~kg} / \mathrm{m}^{2}\right.$, $70.9 \%$ ) with median BMI of $21.5 \mathrm{~kg} / \mathrm{m}^{2}$ before pregnancy. Most couples had college education or higher. Only 3 women smoked during pregnancy and 13 consumed alcohol during pregnancy. However, $31.5 \%$ of mothers were exposed to paternal smoking during pregnancy. Among the newborns, 357 (52.1\%) were boys; $517(75.4 \%)$ were delivered by cesarean section. Mean ( \pm SD) birth weight was $3388 \mathrm{~g}( \pm 469)$ with an average gestational age of 38.8 weeks $( \pm 1.3)$. The proportion of breastfeeding infants in the AD group was $87.3 \%$, which was slightly higher than that in the non-AD group (78.2\%). Univariate analyses show that compared with children without $\mathrm{AD}$, children with $\mathrm{AD}$ were more likely to have a father with older age, family history of allergic disorders and without older siblings.

Among the 811 subjects, 124 had insufficient volume of cord blood for laboratory analyses. The median (Q1Q3) values of PFOA, PFOS, PFNA, PFDA, PFUA, 
Table 1 Characteristics of the study population by childhood atopic dermatitis (AD) status

\begin{tabular}{|c|c|c|c|c|}
\hline & All $(N=687)$ & Non-eczema $(N=514)$ & Eczema $(N=173)$ & $P^{a}$ \\
\hline Prevalence of atopic dermatitis & $173(25.2)$ & & & \\
\hline \multicolumn{5}{|l|}{ Parental characteristics } \\
\hline Maternal age (years) & $29.3 \pm 3.8$ & $29.2 \pm 3.9$ & $29.4 \pm 3.5$ & 0.82 \\
\hline$<25$ & $48(7.0)$ & $38(7.4)$ & $10(5.8)$ & \\
\hline $25-30$ & $347(50.5)$ & $261(50.8)$ & $86(49.7)$ & \\
\hline $30-35$ & $226(32.9)$ & $165(32.1)$ & $61(35.3)$ & \\
\hline$\geq 35$ & $66(9.6)$ & $50(9.7)$ & $16(9.2)$ & \\
\hline Paternal age (years) & $31.7 \pm 4.5$ & $31.5 \pm 4.4$ & $32.1 \pm 4.7$ & 0.12 \\
\hline$<25$ & $15(2.2)$ & $10(2.0)$ & $5(2.9)$ & \\
\hline $25-30$ & $228(33.2)$ & $173(33.7)$ & $55(31.8)$ & \\
\hline $30-35$ & $294(42.8)$ & $229(44.6)$ & $65(37.6)$ & \\
\hline$\geq 35$ & $150(21.8)$ & $102(19.8)$ & $48(27.8)$ & \\
\hline Maternal pre-pregnancy BMI & $21.5 \pm 3.6$ & $21.4 \pm 3.6$ & $21.8 \pm 3.6$ & 0.67 \\
\hline$<18.5$ & $111(16.2)$ & 87 (16.9) & $24(13.9)$ & \\
\hline $18.5-25$ & $487(70.9)$ & $363(70.6)$ & $124(71.7)$ & \\
\hline $25-30$ & $68(9.9)$ & $50(9.7)$ & $18(10.4)$ & \\
\hline$\geq 30$ & $21(3.0)$ & $14(2.7)$ & $7(4.0)$ & \\
\hline \multicolumn{5}{|l|}{ Maternal education } \\
\hline High school or lower & $97(14.1)$ & $78(15.2)$ & $19(11.0)$ & 0.38 \\
\hline College & $529(77.0)$ & $390(75.9)$ & $139(80.3)$ & \\
\hline Postgraduate or higher & $61(8.9)$ & $46(8.9)$ & $15(8.7)$ & \\
\hline \multicolumn{5}{|l|}{ Paternal education } \\
\hline High school or lower & $93(13.5)$ & $70(13.6)$ & $23(13.3)$ & 0.57 \\
\hline College & $503(73.2)$ & $380(73.9)$ & $123(71.1)$ & \\
\hline Postgraduate or higher & $91(13.3)$ & $64(12.5)$ & $27(15.6)$ & \\
\hline \multicolumn{5}{|l|}{ Family history of allergic disorders } \\
\hline Yes & $143(20.8)$ & $94(18.3)$ & $49(28.3)$ & 0.02 \\
\hline No & $533(77.6)$ & $412(80.2)$ & $121(69.9)$ & \\
\hline Unknown & $11(1.6)$ & $8(1.5)$ & $3(1.7)$ & \\
\hline Maternal smoking & & & & 0.31 \\
\hline Yes & $3(0.4)$ & $3(0.6)$ & $0(0)$ & \\
\hline No & $679(99.6)$ & $507(99.4)$ & $172(100)$ & \\
\hline \multicolumn{5}{|l|}{ Parental smoking } \\
\hline Yes & $215(31.5)$ & $167(32.7)$ & $48(28.1)$ & 0.26 \\
\hline No & $467(68.5)$ & $344(67.3)$ & $123(71.9)$ & \\
\hline \multicolumn{5}{|l|}{ Maternal alcohol intake } \\
\hline Yes & $13(1.9)$ & $12(2.3)$ & $1(0.6)$ & 0.15 \\
\hline No & $671(98.1)$ & $501(97.7)$ & $170(99.4)$ & \\
\hline \multicolumn{5}{|l|}{ Newborn characteristics } \\
\hline \multicolumn{5}{|l|}{ Infant sex } \\
\hline Male & $357(52.1)$ & $260(50.6)$ & $97(56.7)$ & 0.16 \\
\hline Female & $328(47.9)$ & $254(49.4)$ & $74(43.3)$ & \\
\hline
\end{tabular}


Table 1 Characteristics of the study population by childhood atopic dermatitis (AD) status (Continued)

\begin{tabular}{|c|c|c|c|c|}
\hline & All $(N=687)$ & Non-eczema $(N=514)$ & Eczema $(N=173)$ & $P^{a}$ \\
\hline Birth weight (g) & $3388 \pm 469$ & $3377 \pm 482$ & $3423 \pm 428$ & 0.52 \\
\hline$<2500$ & $18(2.6)$ & $15(2.9)$ & $3(1.7)$ & \\
\hline $2500-4000$ & $608(88.5)$ & $451(87.7)$ & $157(90.8)$ & \\
\hline$\geq 4000$ & $61(8.9)$ & $48(9.36)$ & $13(7.5)$ & \\
\hline Gestational age (weeks) & $38.8 \pm 1.3$ & $38.8 \pm 1.4$ & $38.8 \pm 1.1$ & 0.18 \\
\hline$\leq 37$ & $54(7.9)$ & $45(8.8)$ & $9(5.2)$ & \\
\hline 38 & $192(28.0)$ & $139(27.0)$ & $53(30.6)$ & \\
\hline 39 & $261(38.0)$ & $187(36.4)$ & $74(42.8)$ & \\
\hline 40 & $137(19.9)$ & $110(21.4)$ & $27(15.6)$ & \\
\hline 41 & $43(6.3)$ & $33(6.4)$ & $10(5.8)$ & \\
\hline \multicolumn{5}{|l|}{ Mode of delivery } \\
\hline Caesarean section & $517(75.4)$ & $389(75.7)$ & $128(74.4)$ & 0.74 \\
\hline Vaginal delivery & $169(24.6)$ & $125(24.3)$ & $44(25.6)$ & \\
\hline \multicolumn{5}{|l|}{ Parity } \\
\hline Nulliparous & $626(91.2)$ & $462(89.9)$ & $164(95.4)$ & 0.03 \\
\hline Parous & $60(8.8)$ & $52(10.1)$ & $8(4.6)$ & \\
\hline \multicolumn{5}{|l|}{ Breastfeeding } \\
\hline Yes & $553(80.5)$ & $402(78.2)$ & $151(87.3)$ & 0.03 \\
\hline No & $28(4.1)$ & $22(4.3)$ & $6(3.5)$ & \\
\hline Unknown & $106(15.4)$ & $90(17.5)$ & $16(9.3)$ & \\
\hline
\end{tabular}

Data presented are $\mathrm{n}(\%)$ or median \pm standard deviation

The missing data: maternal smoking $(n=5)$; paternal smoking $(n=5)$; maternal alcohol intake $(n=3)$; infant sex $(n=2)$; mode of delivery $(n=1)$; parity $(n=1)$

${ }^{a}$ Chi-square test was used to compare the difference of characteristics between Non-AD and AD group

PFDoA, PFHxS and PFBS were 6.98 (4.94-9.55), 2.48 (1.82-3.24), 0.65 (0.50-0.83), $0.36(0.23-0.54), \quad 0.40$ (0.29-0.53), $\quad 0.09 \quad(0.07-0.13), \quad 0.16 \quad(0.13-0.20), \quad 0.05$ (0.04-0.06) $\mathrm{ng} / \mathrm{mL}$, respectively (Table 2). Most PFASs concentrations were slightly but not significantly higher in female than male infants. PFOA concentration in cord blood was higher in children with $\mathrm{AD}$ than in children without $\mathrm{AD}$, while no statistically significant difference in other PFASs was found between those with and without AD (Table 3).

Table 4 shows that the highest PFOA quartile was associated with a 1.7-fold increase in AD risk (OR 1.74, 95\% CI 1.02-2.95) after adjusting for potential confounders, and the second PFDoA quartile was associated with a 1.8fold increase in AD risk (OR 1.83, 95\% CI 1.09-3.08). The significant modification effect of sex on the associations of interest was found only for the association between PFHxS exposure and AD. Thus, the effects of PFASs on AD were generally similar in male and female children.

Table 5 shows that overall, prenatal exposures to PFOA, PFDA, PFDoA and PFHxS were significantly associated with childhood $\mathrm{AD}$ in girls during the first 24 months (Table 5). In the first fully adjusted model, a log-unit increase in PFOA was associated with a 2.1-fold increase in $\mathrm{AD}$ risk (AOR 2.07, 95\% CI 1.13-3.80). The corresponding number for PFNA was 2.22 (1.07-4.58). In the second fully adjusted model, when PFASs were grouped by quartiles, AOR increased with increasing PFOA and PFNA levels. The strongest associations were found in Q4. Specially, the highest quartiles of PFOA, PFNA, PFDA and PFHxS were associated with AD with AOR (95\% CI) being 2.52 (1.12-5.68), 2.14 (0.97-4.74), 2.14 (1.00-4.57), and 2.30 (1.03-5.15), respectively. Additionally, the second quartile of perfluorododecanoic acid (PFDoA) was associated with a 3.2-fold increase in AD risk $(3.24,1.44-7.27)$. Due to small sample size, the associations between AD with prenatal exposure to PFNA were close to statistical significance. No other PFASs were significantly associated with $\mathrm{AD}$ in girls. However, no significant associations were found between PFASs and AD in boys (Additional file 2: Table S2).

\section{Discussion}

Our prospective cohort study suggests that prenatal PFASs exposures may increase the risk of childhood AD. In particular, we found that PFOA, PFDA, PFDoA and PFHxS in cord blood increased the risk of childhood AD in girls only. In addition, the associations between AD with prenatal exposure to PFNA were close to statistical significance. 
Table 2 Concentrations $(\mathrm{ng} / \mathrm{mL}$ ) of the most prevalent PFASs in cord plasma samples of this study population

\begin{tabular}{|c|c|c|c|c|c|c|c|c|}
\hline & PFOS & PFOA & PFNA & PFDA & PFUA & PFDoA & PFHXS & PFBS \\
\hline \multicolumn{9}{|l|}{ All $(n=687)$} \\
\hline N > LOD (\%) & $687(100)$ & $686(99.9)$ & $687(100)$ & 681 (99.1) & $686(99.9)$ & $621(90.4)$ & $687(100)$ & $668(97.2)$ \\
\hline Range & $0.39-65.61$ & $<$ LOD-29.97 & $0.18-3.29$ & $<\mathrm{LOD}-5.73$ & $<\mathrm{LOD}-5.27$ & $<\mathrm{LOD}-1.14$ & $0.05-0.85$ & $<\mathrm{LOD}-0.46$ \\
\hline Mean (SD) & $2.93(3.11)$ & 7.73 (3.98) & $0.70(0.29)$ & $0.45(0.42)$ & $0.46(0.34)$ & $0.10(0.07)$ & $0.18(0.08)$ & $0.05(0.03)$ \\
\hline Median & 2.48 & 6.98 & 0.64 & 0.36 & 0.4 & 0.09 & 0.16 & 0.05 \\
\hline \multicolumn{9}{|l|}{ Female $(n=328)$} \\
\hline N > LOD (\%) & $328(100)$ & $328(100)$ & $328(100)$ & 324 (98.8) & 327 (99.7) & $291(88.7)$ & $328(100)$ & $318(97.0)$ \\
\hline Range & 0.39-18.68 & $0.70-29.97$ & $0.18-3.29$ & $<\mathrm{LOD}-2.51$ & $<\mathrm{LOD}-2.60$ & $<\mathrm{LOD}-0.51$ & $0.07-0.78$ & $<$ LOD-0.39 \\
\hline Mean (SD) & $2.81(1.86)$ & 7.57 (3.71) & $0.71(0.31)$ & $0.44(0.32)$ & $0.45(0.26)$ & $0.11(0.05)$ & $0.17(0.08)$ & $0.05(0.03)$ \\
\hline Median & 2.47 & 7 & 0.66 & 0.38 & 0.41 & 0.1 & 0.16 & 0.05 \\
\hline \multicolumn{9}{|l|}{ Male $(n=357)$} \\
\hline N > LOD (\%) & $357(100)$ & 356 (99.7) & $357(100)$ & 355 (99.4) & $357(100)$ & 328 (91.9) & $359(100)$ & 348 (97.5) \\
\hline Range & $0.62-65.61$ & $<$ LOD-25.99 & $0.18-2.04$ & $<\mathrm{LOD}-5.73$ & $0.05-5.27$ & $<$ LOD-1.14 & $0.05-0.85$ & $<\mathrm{LOD}-0.46$ \\
\hline Mean (SD) & 3.04 (3.93) & $7.86(4.22)$ & $0.69(0.28)$ & $0.45(0.50)$ & $0.46(0.40)$ & $0.10(0.08)$ & $0.18(0.08)$ & $0.05(0.03)$ \\
\hline Median & 2.49 & 6.89 & 0.64 & 0.35 & 0.39 & 0.09 & 0.16 & 0.05 \\
\hline
\end{tabular}

$\angle O D$ limit of detection, $S D$ standard deviation, $C l$ confidence intervals

LOD (ng/mL): PFOA (0.09), PFOS (0.09), PFNA (0.02), PFDA (0.02), PFUA (0.02), PFDoA (0.05), PFHxS (0.02), PFBS (0.009)

Five studies have reported the association between prenatal exposure and childhood $\mathrm{AD}$, but the results were inconsistent. In a cohort study conducted in Taiwan, the highest quartile of PFOS appeared to be positively associated with $\mathrm{AD}$ with an OR of 2.79 (95\% CI: $1.06,7.38)$ [25]. However, after adjusted by potential confounders, the association was no longer statistically significant. Another two birth cohort studies from Norway and Japan found no significant associations between PFOA, PFOS, PFNA and PFHxS and AD or eczema in early childhood [26, 27]. Likewise, a birth cohort study in 1024 infants conducted in Greenland and Ukraine, reported that principal component (PC) score, dominated by PFOA, was not associated with eczema [28]. In contrast, a birth cohort study in Japan

Table 3 Cord blood concentrations of PFASs ( $\mathrm{ng} / \mathrm{mL}$ ) by childhood atopic dermatitis (AD) status

\begin{tabular}{llll}
\hline PFAS & Non-AD $(N=514)$ & $A D(N=173)$ & $P$ \\
\hline PFOS & $2.46(1.80-3.17)$ & $2.54(1.83-3.37)$ & 0.29 \\
PFOA & $6.76(4.84-9.29)$ & $7.17(5.22-10.19)$ & 0.04 \\
PFNA & $0.64(0.49-0.81)$ & $0.66(0.54-0.86)$ & 0.09 \\
PFDA & $0.36(0.23-0.52)$ & $0.39(0.26-0.58)$ & 0.13 \\
PFUA & $0.40(0.29-0.52)$ & $0.41(0.30-0.57)$ & 0.29 \\
PFDoA & $0.09(0.07-0.13)$ & $0.09(0.07-0.12)$ & 0.87 \\
PFHXS & $0.16(0.13-0.20)$ & $0.16(0.14-0.21)$ & 0.16 \\
PFBS & $0.05(0.04-0.06)$ & $0.05(0.04-0.06)$ & 0.43 \\
\hline
\end{tabular}

Abbreviations: PFOS Perfluorooctane sulfonate, PFNA Perfluorononanoic acid, PFDA Perfluorodecanoic acid, PFUA Perfluoroundecanoic acid, PFHXS Perfluorohexanesulfonate, $P F O A$ Perfluorooctanate, PFDoDA Perfluorododecanoic acid, PFBS Perfluorobutane sulfonate involving 2063 subjects found that prenatal exposure to perfluorotridecanoic acid (PFTrDA) was associated with a decreased risk of developing eczema in female infants at 24 months $(\mathrm{OR}=0.39$; 95\% CI: $0.23,0.64)$ [29]. After following for another 2 years, PFOS levels in the highest quartile were associated with an increased risk of total infectious diseases in all children $(\mathrm{OR}=1.61 ; 95 \% \mathrm{CI}$ : $1.18,2.21$ ), while the highest quartile of PFHxS was associated with a higher risk of total infectious diseases only among girls (OR $=1.55,95 \%$ CI: 0.976, 2.45) [30]. These studies provided evidence for the potential long-lasting effect of PFASs on children's immune system.

Several studies also reported associations between PFASs and other allergic disorders in children and adults. A US national study found that serum PFOA was positively associated with asthma $(\mathrm{OR}=1.18,95 \% \mathrm{CI}$ : $1.01,1.39$ ) in adolescents aged $12-19$ years, while PFOS was inversely associated with both asthma and wheezing $(\mathrm{OR}=0.88 ; 95 \% \mathrm{CI}: 0.74,1.04$, and $\mathrm{OR}=0.83$; 95\% CI: $0.67,1.02$, respectively) [15]. In addition, a case-control study with 231 asthmatic and 225 non-asthmatic children aged 10-15 years in Taiwan found that PFOA and PFOS were associated with asthma in a dose-response pattern [14].

The mechanisms of prenatal PFASs exposure on the development of AD have not been fully understood. Animal and human studies suggest that PFOA and PFOS may increase IgE levels in blood. For example, PFASs concentrations in cord blood were positively associated with cord blood IgE levels in Taiwan infants (per ln-unit: $\beta=0.134 \mathrm{KU} / \mathrm{l}, p=0.047$ for PFOA; $\beta=0.161 \mathrm{KU} / \mathrm{l}, p=$ 0.017 for PFOS) [25]. Furthermore, both animal and 
Table 4 Association between cord blood concentrations of PFASs and risk for childhood AD ( $n=687$ )

\begin{tabular}{|c|c|c|c|c|c|}
\hline PFASs & Number & Number of cases & Crude OR (95\%Cl) & Adjusted OR (95\%Cl) & p for sex-PFASs interaction \\
\hline \multicolumn{6}{|l|}{ PFOS } \\
\hline Continuous $^{\mathrm{a}}$ & 687 & 173 & $1.17(0.85-1.62)$ & $1.23(0.85-1.76)$ & 0.33 \\
\hline $\mathrm{Q} 1(<1.81)^{\mathrm{b}}$ & 171 & 40 & 1 & 1 & 0.26 \\
\hline Q2 (1.81-2.48) & 172 & 42 & $1.06(0.64-1.74)$ & $0.93(0.56-1.58)$ & \\
\hline Q3 (2.48-3.24) & 172 & 41 & $1.03(0.62-1.69)$ & $1.00(0.59-1.70)$ & \\
\hline Q4 ( $\geq 3.24)$ & 172 & 50 & $1.34(0.83-2.18)$ & $1.31(0.78-2.20)$ & \\
\hline$p$ for trend ${ }^{c}$ & & & 0.27 & 0.27 & \\
\hline \multicolumn{6}{|l|}{ PFOA } \\
\hline Continuous $^{\mathrm{a}}$ & 687 & 173 & $1.44(1.02-2.03)$ & $1.35(0.93-1.97)$ & 0.17 \\
\hline Q1 $(<4.94)^{\mathrm{b}}$ & 171 & 34 & 1 & 1 & 0.14 \\
\hline Q2 (4.94-6.98) & 172 & 46 & $1.47(0.89-2.44)$ & $1.48(0.87-2.52)$ & \\
\hline Q3 (6.98-9.55) & 172 & 39 & $1.18(0.70-1.98)$ & $1.16(0.67-2.00)$ & \\
\hline Q4 ( $\geq 9.55)$ & 172 & 54 & $1.84(1.12-3.02)$ & $1.74(1.02-2.95)$ & \\
\hline$p$ for trend ${ }^{c}$ & & & 0.04 & 0.1 & \\
\hline \multicolumn{6}{|l|}{ PFNA } \\
\hline Continuous $^{\mathrm{a}}$ & 687 & 173 & $1.49(0.95-2.32)$ & $1.53(0.94-2.47)$ & 0.46 \\
\hline Q1 $(<0.50)^{b}$ & 171 & 36 & 1 & 1 & 0.33 \\
\hline Q2 (0.50-0.64) & 172 & 44 & $1.29(0.78-2.13)$ & $1.15(0.67-1.97)$ & \\
\hline Q3 (0.64-0.83) & 172 & 44 & $1.29(0.78-2.13)$ & $1.20(0.71-2.05)$ & \\
\hline Q4 ( $\geq 0.83)$ & 172 & 49 & $1.49(0.91-2.45)$ & $1.47(0.87-2.50)$ & \\
\hline$p$ for trend ${ }^{c}$ & & & 0.13 & 0.15 & \\
\hline \multicolumn{6}{|l|}{ PFDA } \\
\hline Continuous $^{\mathrm{a}}$ & 687 & 173 & $1.18(0.93-1.50)$ & $1.22(0.94-1.58)$ & 0.65 \\
\hline Q1 $(<0.23)^{\mathrm{b}}$ & 171 & 40 & 1 & 1 & 0.68 \\
\hline Q2 (0.23-0.36) & 172 & 36 & $0.87(0.52-1.44)$ & $0.94(0.55-1.60)$ & \\
\hline Q3 (0.36-0.54) & 172 & 45 & $1.16(0.71-1.90)$ & $1.15(0.68-1.95)$ & \\
\hline Q4 ( $\geq 0.54)$ & 172 & 52 & $1.42(0.88-2.30)$ & $1.58(0.94-2.65)$ & \\
\hline$p$ for trend ${ }^{c}$ & & & 0.08 & 0.06 & \\
\hline \multicolumn{6}{|l|}{ PFUA } \\
\hline Continuous $^{\mathrm{a}}$ & 687 & 173 & $1.18(0.86-1.63)$ & $1.24(0.88-1.75)$ & 0.67 \\
\hline Q1 $(<0.29)^{\mathrm{b}}$ & 171 & 40 & 1 & 1 & 0.83 \\
\hline Q2 (0.29-0.40) & 172 & 40 & $0.99(0.60-1.64)$ & $1.01(0.59-1.71)$ & \\
\hline Q3 (0.40-0.53) & 172 & 44 & $1.13(0.69-1.84)$ & $1.13(0.67-1.90)$ & \\
\hline Q4 ( $\geq 0.53)$ & 172 & 49 & $1.31(0.80-2.12)$ & $1.36(0.81-2.28)$ & \\
\hline$p$ for trend ${ }^{c}$ & & & 0.23 & 0.22 & \\
\hline \multicolumn{6}{|l|}{ PFDoA } \\
\hline Continuous $^{a}$ & 687 & 173 & $0.96(0.70-1.30)$ & $1.00(0.72-1.39)$ & 0.28 \\
\hline Q1 $(\leq 0.069)^{\mathrm{b}}$ & 171 & 36 & 1 & 1 & 0.76 \\
\hline Q2 (0.069-0.094) & 172 & 54 & $1.72(1.05-2.80)$ & $1.83(1.09-3.08)$ & \\
\hline Q3 (0.094-0.125) & 172 & 42 & $1.21(0.73-2.01)$ & $1.37(0.80-2.34)$ & \\
\hline $\mathrm{Q} 4(\geq 0.125)$ & 172 & 41 & $1.17(0.71-1.95)$ & $1.18(0.68-2.04)$ & \\
\hline$p$ for trend ${ }^{c}$ & & & 0.93 & 0.87 & \\
\hline
\end{tabular}


Table 4 Association between cord blood concentrations of PFASs and risk for childhood AD ( $n=687)$ (Continued)

\begin{tabular}{|c|c|c|c|c|c|}
\hline PFASs & Number & Number of cases & Crude OR $(95 \% \mathrm{Cl})$ & Adjusted OR (95\%Cl) & $p$ for sex-PFASs interaction \\
\hline \multicolumn{6}{|l|}{$\mathrm{PFHXS}$} \\
\hline Continuous $^{\mathrm{a}}$ & 687 & 173 & $1.31(0.80-2.13)$ & $1.08(0.62-1.85)$ & 0.43 \\
\hline $\mathrm{Q} 1(\leq 0.13)^{\mathrm{b}}$ & 171 & 37 & 1 & 1 & 0.05 \\
\hline Q2 (0.13-0.16) & 172 & 43 & $1.21(0.73-1.99)$ & $1.25(0.74-2.12)$ & \\
\hline Q3 (0.16-0.20) & 172 & 46 & $1.32(0.81-2.17)$ & $1.15(0.68-1.94)$ & \\
\hline Q4 ( $\geq 0.20)$ & 172 & 47 & $1.36(0.83-2.23)$ & $1.14(0.67-1.94)$ & \\
\hline$p$ for trend ${ }^{c}$ & & & 0.2 & 0.73 & \\
\hline \multicolumn{6}{|l|}{ PFBS } \\
\hline Continuous $^{\mathrm{a}}$ & 687 & 173 & $1.20(0.87-1.66)$ & $1.22(0.87-1.72)$ & 0.83 \\
\hline Q1 $(\leq 0.037)^{\mathrm{b}}$ & 170 & 39 & 1 & 1 & 0.33 \\
\hline Q2 (0.037-0.047) & 171 & 43 & $1.13(0.69-1.86)$ & $1.28(0.75-2.17)$ & \\
\hline Q3 (0.047-0.062) & 174 & 46 & $1.21(0.74-1.97)$ & $1.18(0.70-2.00)$ & \\
\hline $\mathrm{Q} 4(\geq 0.062)$ & 172 & 45 & $1.19(0.73-1.95)$ & $1.15(0.68-1.95)$ & \\
\hline$p$ for trend ${ }^{c}$ & & & 0.46 & 0.70 & \\
\hline \multicolumn{6}{|c|}{$\begin{array}{l}\text { Models were adjusted for all the confounders foll } \\
\text { (continuous), birth weight (categorical), maternal } \\
\text { history of allergic disorders (categorical), infant se } \\
\text { breastfeeding (categorical) } \\
\text { a Log-transformed PFASs as continuous variables } \\
\text { b Reference category }\end{array}$} \\
\hline
\end{tabular}

human studies suggested that PFOS may increase Th2 cytokines secretion (IL-4 and IL-10) and decrease Th1 cytokines (IL-2 and IFN- $\gamma$ ) at doses comparable to those in humans, shifting immune response toward Th2 state [31, 32]. A previous study showed that PFOA, PFOS and PFDA suppressed LPS-induced TNF- $\alpha$ production; PFOS and PFDA inhibited PHA-induced IL-10 release in human peripheral blood leukocytes (hPBL) and human promyelocytic cell line THP-1 [33].

Oxidative stress might be another potential mechanism of the immunotoxic effects of PFASs. Wielsøe et al. exposed human hepatoma cell line (HepG2) with PFHxS, PFOA, PFOS and PFNA for $24 \mathrm{~h}$ and found a dose-dependent increase in DNA damage and increased ROS generation [34]. PFNA was also found to have immunomodulatory effects on leukocyte populations and immune function, including decreased spleen size and a decreased ratio of CD4 +/CD8+ double-positive population in thymus of mice [35].

In addition, peroxisome proliferator activated receptors (PPARs) signaling pathway might also play a role in the effects of PFASs on childhood AD. Previous study showed that PFOA and PFOS significantly increased PPAR- $\alpha$ and PPAR- $\gamma$ in human cells [36], which, in turn, could shift cytokine secretion by inhibiting IFN- $\gamma$ and promoting IL-4 secretion in human T-cell lines [37, 38]. The changes of these cytokines resulted from PFASs exposure might impair the immune system and immune responses, especially in young children when the immune system is still developing.
Nonetheless, a prospective cohort study in Japan found an inverse association between maternal PFOS concentration and cord blood IgE levels among female infants $(\beta=$ -3.08 , 95\%CI: $-5.43,-0.73$ ) [26]. Thus, the evidence is far from consistent, and our understanding of the underlying biological mechanisms is still incomplete.

A birth cohort study conducted in Japan between 2002 and 2005, showed that cortisol and cortisone concentrations were significantly lower in cord blood of infants with prenatal PFOS exposure in Q4 compared with those in Q1 $(\beta=-23.98 \mathrm{ng} / \mathrm{mL}, 95 \% \mathrm{CI}:-47.12,-11.99$ for cortisol; $\beta=-63.21 \mathrm{ng} / \mathrm{mL}, 95 \% \mathrm{CI}:-132.56,-26.72$ for cortisone). Meanwhile, the highest quartile of prenatal PFOS exposure was positively associated with a significant higher DHEA level ( $\beta=1.33 \mathrm{ng} / \mathrm{mL}, 95 \%$ CI: $0.17,1.82)$, whereas PFOA showed a negative association with DHEA levels $(\beta$ $=-1.23 \mathrm{ng} / \mathrm{mL}, 95 \% \mathrm{CI}:-1.72,-0.25)$ [39]. In addition, animal and human data suggested that perinatal exposure to glucocorticoid may program the fetal hypothalamicpituitary-adrenal (HPA) axis affecting its development, resulting in long-lasting effect in HPA axis function $[40,41]$. These findings revealed that prenatal exposure may have an effect on the balance of steroid hormones, which was one of the potential mechanisms of the immunotoxical effects of PFASs [42].

Our study discovered that the association between prenatal exposure to PFASs and childhood AD existed only in girls, but the biologic mechanism is unclear. A previous study showed that exposure to higher levels of 
Table 5 Association between cord blood concentrations of PFASs and risk for childhood AD in female children $(n=328)$

\begin{tabular}{|c|c|c|c|c|}
\hline PFASS & Number & Number of cases & Crude OR $(95 \% \mathrm{Cl})$ & Adjusted OR (95\%Cl) \\
\hline \multicolumn{5}{|l|}{ PFOS } \\
\hline Continuous $^{a}$ & 328 & 74 & $1.05(0.64-1.71)$ & $1.10(0.64-1.87)$ \\
\hline Q1 $(<1.80)^{\mathrm{b}}$ & 82 & 21 & 1 & 1 \\
\hline Q2 (1.80-2.47) & 82 & 16 & $0.70(0.34-1.47)$ & $0.73(0.33-1.61)$ \\
\hline Q3 (2.47-3.22) & 82 & 16 & $0.70(0.34-1.47)$ & $0.71(0.32-1.60)$ \\
\hline Q4 ( $\geq 3.22)$ & 82 & 21 & $1.00(0.50-2.02)$ & $1.08(0.50-2.35)$ \\
\hline$p$ for trend ${ }^{c}$ & & & 0.85 & 0.74 \\
\hline \multicolumn{5}{|l|}{ PFOA } \\
\hline Continuous $^{a}$ & 328 & 74 & $2.05(1.17-3.61)$ & $2.07(1.13-3.80)$ \\
\hline Q1 $(<4.94)^{\mathrm{b}}$ & 82 & 13 & 1 & 1 \\
\hline Q2 (4.94-7.00) & 82 & 14 & $1.09(0.48-2.50)$ & $1.23(0.52-2.93)$ \\
\hline Q3 (7.00-9.42) & 82 & 20 & $1.71(0.79-3.73)$ & $1.81(0.79-4.14)$ \\
\hline Q4 ( $\geq 9.42)$ & 82 & 27 & $2.61(1.23-5.52)$ & $2.52(1.12-5.68)$ \\
\hline$p$ for trend ${ }^{c}$ & & & 0.004 & 0.01 \\
\hline \multicolumn{5}{|l|}{ PFNA } \\
\hline Continuous $^{\mathrm{a}}$ & 328 & 74 & $1.92(0.99-3.74)$ & $2.22(1.07-4.58)$ \\
\hline Q1 $(<0.50)^{\mathrm{b}}$ & 82 & 15 & 1 & 1 \\
\hline Q2 (0.50-0.66) & 82 & 15 & $1.00(0.45-2.21)$ & $1.10(0.46-2.62)$ \\
\hline Q3 (0.66-0.85) & 82 & 19 & $1.35(0.63-2.88)$ & $1.30(0.57-2.97)$ \\
\hline Q4 ( $\geq 0.85)$ & 82 & 25 & $1.96(0.94-4.07)$ & $2.14(0.97-4.74)$ \\
\hline$p$ for trend ${ }^{c}$ & & & 0.04 & 0.05 \\
\hline \multicolumn{5}{|l|}{ PFDA } \\
\hline Continuous $^{\mathrm{a}}$ & 328 & 74 & $1.14(0.80-1.63)$ & $1.25(0.86-1.84)$ \\
\hline Q1 $(<0.25)^{\mathrm{b}}$ & 82 & 19 & 1 & 1 \\
\hline Q2 (0.25-0.38) & 82 & 17 & $0.87(0.41-1.82)$ & $1.01(0.46-2.23)$ \\
\hline Q3 (0.38-0.53) & 82 & 12 & $0.57(0.26-1.26)$ & $0.54(0.22-1.30)$ \\
\hline Q4 ( $\geq 0.53)$ & 82 & 26 & $1.54(0.77-3.08)$ & $2.14(1.00-4.57)$ \\
\hline$p$ for trend ${ }^{c}$ & & & 0.35 & 0.13 \\
\hline \multicolumn{5}{|l|}{ PFUA } \\
\hline Continuous $^{a}$ & 328 & 74 & $1.15(0.71-1.86)$ & $1.24(0.74-2.10)$ \\
\hline Q1 $(<0.29)^{\mathrm{b}}$ & 82 & 17 & 1 & 1 \\
\hline Q2 (0.29-0.41) & 82 & 17 & $1.00(0.47-2.13)$ & $1.11(0.49-2.50)$ \\
\hline Q3 (0.41-0.54) & 82 & 19 & $1.15(0.55-2.42)$ & $1.20(0.55-2.64)$ \\
\hline Q4 ( $\geq 0.54)$ & 82 & 21 & $1.32(0.64-2.73)$ & $1.55(0.70-3.43)$ \\
\hline$p$ for trend ${ }^{c}$ & & & 0.41 & 0.28 \\
\hline \multicolumn{5}{|l|}{ PFDoA } \\
\hline Continuous $^{\mathrm{a}}$ & 328 & 74 & $0.88(0.57-1.36)$ & $0.91(0.57-1.46)$ \\
\hline $\mathrm{Q} 1(\leq 0.069)^{\mathrm{b}}$ & 82 & 13 & 1 & 1 \\
\hline Q2 (0.069-0.096) & 82 & 27 & $2.61(1.23-5.52)$ & $3.24(1.44-7.27)$ \\
\hline Q3 (0.096-0.128) & 82 & 17 & $1.39(0.63-3.08)$ & $1.44(0.62-3.36)$ \\
\hline $\mathrm{Q} 4(\geq 0.128)$ & 82 & 17 & $1.39(0.63-3.08)$ & $1.63(0.68-3.92)$ \\
\hline$p$ for trend $d^{c}$ & & & 0.91 & 0.74 \\
\hline
\end{tabular}


Table 5 Association between cord blood concentrations of PFASs and risk for childhood AD in female children ( $n=328)($ Continued)

\begin{tabular}{|c|c|c|c|c|}
\hline PFASs & Number & Number of cases & Crude OR $(95 \% \mathrm{Cl})$ & Adjusted OR (95\%Cl) \\
\hline \multicolumn{5}{|l|}{ PFHXS } \\
\hline Continuous $^{a}$ & 328 & 74 & $1.59(0.77-3.31)$ & $1.43(0.64-3.15)$ \\
\hline $\mathrm{Q} 1(\leq 0.13)^{\mathrm{b}}$ & 82 & 14 & 1 & 1 \\
\hline Q2 (0.13-0.16) & 82 & 16 & $1.18(0.53-2.60)$ & $1.43(0.62-3.30)$ \\
\hline Q3 (0.16-0.20) & 82 & 18 & $1.37(0.63-2.97)$ & $1.29(0.55-2.99)$ \\
\hline Q4 ( $\geq 0.20)$ & 82 & 26 & $2.26(1.08-4.73)$ & $2.30(1.03-5.15)$ \\
\hline$p$ for trend ${ }^{c}$ & & & 0.03 & 0.06 \\
\hline \multicolumn{5}{|l|}{ PFBS } \\
\hline Continuous $^{\mathrm{a}}$ & 328 & 74 & $1.22(0.75-1.97)$ & $1.23(0.74-2.04)$ \\
\hline Q1 $(\leq 0.037)^{\mathrm{b}}$ & 82 & 14 & 1 & 1 \\
\hline Q2 (0.037-0.047) & 81 & 18 & $1.39(0.64-3.02)$ & $1.65(0.70-3.84)$ \\
\hline Q3 (0.047-0.061) & 83 & 23 & $1.86(0.88-3.94)$ & $1.83(0.81-4.17)$ \\
\hline Q4 ( $\geq 0.061)$ & 82 & 19 & $1.46(0.68-3.17)$ & $1.50(0.65-3.48)$ \\
\hline$p$ for trend ${ }^{c}$ & & & 0.25 & 0.35 \\
\hline
\end{tabular}

Models were adjusted for all the confounders followed, maternal age (continuous), maternal pre-pregnancy BMI (continuous), gestational week at delivery (continuous), birth weight (categorical), maternal education (categorical), paternal education (categorical), parity (categorical), mode of delivery (categorical), family history of allergic disorders (categorical), family income (categorical), maternal ethnicity (categorical), paternal smoking (categorical) and breastfeeding (categorical)

aLog-transformed PFASs as continuous variables

${ }^{\mathrm{b}}$ Reference category

$c_{p}$-Values for exposure were modeled according to the median value of each quartile

PFASs resulted in lower testosterone and higher estradiol levels, and more significant associations of PFASs with reproductive hormones were found in male adolescents than in females [43]. This effect was supported by an experimental study conducted in mice [44]. A casecontrol study reported that PFASs were positively associated with estradiol levels and negatively associated with testosterone levels among asthmatics [45]. Furthermore, the interactions between estradiol and PFASs were significant for PFOS $(p=0.026)$ and PFNA $(p=$ 0.043 ) among girls, suggesting that reproductive hormones might amplify the association between PFASs and asthma.

The PFOA level in our study was higher than that reported in other countries and was the most abundant among all the PFAS members [24]. The higher PFAS levels in Shanghai may be due to higher levels of pollutants in Yangtze river, probably because there are many fluoropolymer factories in the Yangtze Delta. For example, PFAS levels in tap water in Shanghai was higher than that in 19 cities in China, Japan, India, the USA, and Canada between 2006 and 2008 [46].

Our study also showed a very high cesarean section rate. This is consistent with the situation in China where cesarean delivery on maternal request accounts for nearly $50 \%$ of all cesarean sections [47]. The effect of cesarean section on AD has not been well studied and the results were inconclusive. The effect of cesarean section on AD during the first 3 years of life was not significant (OR 1.35; 95\% CI 0.74-2.47) in a birth cohort study
[48]. The OR of having AD in adolescents aged from 12 to 18 years born by caesarean section compared with vaginal delivery was 1.50 (95\% CI 1.01-2.22) in a crosssectional study [49]. However, our study did not show any association (OR 0.94, 95\% CI 0.63-1.39).

The prospective cohort study design, the AD diagnosis confirmed by two dermatologists, and the reliable laboratory assessments were strengths of our study. We also adjusted for a number of potential confounders in the multivariable models. On the other hand, it is worth noting that $23.2 \%$ of children were excluded from analyses due to missing data or loss to follow-up, resulting in reduced statistical power for sex-stratified analyses. However, there were no significant differences in basic characteristics between those remained in analyses and those excluded. In addition, our study is still not large enough to examine some PFASs with a wider variation of exposure and AD. Finally, unmeasured confounding is possible and residual confounding could not be ruled out.

\section{Conclusion}

Prenatal exposure to PFOA, PFDA, PFDoA and PFHxS was positively associated with childhood AD in girls during the first 24 months of life. In addition, the associations between $\mathrm{AD}$ with prenatal exposure to PFNA were close to statistical significance. Future studies are warranted to investigate the underlying mechanisms of the effects of PFASs and allergic diseases in early childhood. 


\section{Additional files}

Additional file 1: Figure S1. Direct Acyclic Graph (DAG) of the association between prenatal PFASs exposure and childhood AD. (DOCX $305 \mathrm{~kb}$ )

Additional file 2: Table S1. Characteristics of the study population between the current study and total population. Table S2. Association between cord blood concentrations of PFASs and risk for childhood AD in male children $(n=359)$. (DOCX $23 \mathrm{~kb})$

\section{Abbreviations}

AD: Atopic dermatitis; Cl: Confidence interval; ESI: Electrospray ionization; HPLC: High performance liquid chromatography; ISAAC: International Study of Asthma and Allergies in Childhood; MRM: Multiple reaction monitoring; OR: Odds ratio; PFASs: Perfluoroalkyl and polyfluoroalkyl substances; PFBS: Perfluorobutane sulfonic acid; PFDA: Perfluorodecanoic acid; PFDoA: Perfluorododecanoic acid; PFHpA: Perfluoroheptanoic acid; PFHXS: Perfluorohexane sulfonic acid; PFNA: Perfluorononanoic acid; PFOA: Perfluorooctanoic acid; PFOS: Perfluorooctane sulfonate;

PFOSA: Perfluorooctane sulfonamide; PFUA: Perfluoroundecanoic acid

\section{Acknowledgements}

The authors express their thanks to all the participants of birth cohort study and the workers in the Ministry of Education-Shanghai Key Laboratory of Children's Environmental Health.

\section{Funding}

This study was supported in part by grants from Ministry of Science and Technology of China (2014CB943300) and National Natural Science Foundation of China $(81273091 ; 81402686)$. This study was also supported in part by the Shanghai Municipal Health and Family Planning Commission (15GWZK0401) and Shanghai Science and Technology Commission (14XD1403300).

\section{Availability of data and materials}

The datasets analyzed in this study are available from the corresponding author zhangjun@xinhuamed.com.cn on reasonable request.

\section{Authors' contributions}

QC and JZ designed the study. QC analyzed the data, and drafted the manuscript. $\mathrm{RH}$ extensively edited the manuscript. QC, LsH, YZ and XW collected the data. QC, LsH and YZ performed experiments. LH and YG diagnosed all the participants in the present study. All authors provided critical comments and input in the paper. JZ takes the overall responsibility for the study. All authors have reviewed the final version of the manuscript and agreed to the submission. All authors read and approved the final manuscript.

\section{Ethics approval and consent to participate}

The ethics approval was obtained from the Xinhua Hospital and International Peace Maternity and Infant Health affiliated to the Shanghai Jiao Tong University School of Medicine. This study was conducted in accordance with the principles of the Helsinki Declaration.

\section{Consent for publication}

Not applicable.

\section{Competing interests}

The authors declare that they have no competing interests.

\section{Publisher's Note}

Springer Nature remains neutral with regard to jurisdictional claims in published maps and institutional affiliations.

\section{Author details}

${ }^{1}$ Ministry of Education-Shanghai Key Laboratory of Children's Environmental Health, Xinhua Hospital, Shanghai Jiao Tong University School of Medicine, 1665 Kongjiang Road, Shanghai 200092, China. ${ }^{2}$ Department of Pediatric Pulmonology, Xinhua Hospital, Shanghai Jiao Tong University School of Medicine, Shanghai 200092, China. ${ }^{3}$ Department of Dermatology, Xinhua Hospital, Shanghai Jiao Tong University School of Medicine, Shanghai 200092, China. ${ }^{4}$ Department of Pediatrics, Xinhua Hospital, Shanghai Jiao
Tong University School of Medicine, Shanghai 200092, China. ${ }^{5}$ Clinical research unit, Xinhua Hospital, Shanghai Jiao Tong University School of Medicine, Shanghai 200092, China. 'Department of Child Health Care, Shanghai Children's Hospital, Shanghai Jiao Tong University School of Medicine, Shanghai 200040, China.

Received: 25 July 2017 Accepted: 5 January 2018

Published online: 17 January 2018

\section{References}

1. Lehmler H-J. Synthesis of environmentally relevant fluorinated surfactants-a review. Chemosphere. 2005;58(11):1471-96

2. Houde MMJ, Letcher RJ, Solomon KR, Muir DC. Biological monitoring of polyfluoroalkyl substances: a review. Environ Sci Technol. 2006;40(11):3463-73.

3. Olsen GW, Burris JM, Ehresman DJ, Froehlich JW, Seacat AM, Butenhoff JL, Zobel LR. Half-life of serum elimination of Perfluorooctanesulfonate, Perfluorohexanesulfonate, and Perfluorooctanoate in retired Fluorochemical production workers. Environ Health Perspect. 2007;115(9):1298-305.

4. Kato K, Wong L-Y, Jia LT, Kuklenyik Z, Calafat AM. Trends in exposure to Polyfluoroalkyl Chemicals in the U.S. population: 1999-2008. Environ Sci Technol. 2011:45(19):8037-45

5. Glynn A, Berger U, Bignert A, Ullah S, Aune M, Lignell S, Darnerud PO. Perfluorinated alkyl acids in blood serum from Primiparous women in Sweden: serial sampling during pregnancy and nursing, and tempora trends 1996-2010. Environ Sci Technol. 2012;46(16):9071-9.

6. Li L, Zhai Z, Liu J, Hu J. Estimating industrial and domestic environmental releases of perfluorooctanoic acid and its salts in China from 2004 to 2012. Chemosphere. 2015;129:100-9.

7. Lau C, Butenhoff JL, Rogers JM. The developmental toxicity of perfluoroalkyl acids and their derivatives. Toxicol Appl Pharmacol. 2004;198(2):231-41.

8. Lau C, Anitole K, Hodes C, Lai D, Pfahles-Hutchens A, Seed J. Perfluoroalkyl acids: a review of monitoring and toxicological findings. Toxicol Sci. 2007:99(2):366-94

9. Olsen GW, Butenhoff JL, Zobel LR. Perfluoroalkyl chemicals and human fetal development: an epidemiologic review with clinical and toxicological perspectives. Reprod Toxicol. 2009;27(3-4):212-30.

10. Corsini E, Avogadro A, Galbiati V, dell'Agli M, Marinovich M, Galli CL, Germolec DR. In vitro evaluation of the immunotoxic potential of perfluorinated compounds (PFCs). Toxicol Appl Pharmacol. 2011;250(2):108-16.

11. Halsne R, Tandberg J, Lobert V, Østby G, Thoen E, Ropstad E, Verhaegen S Effects of perfluorinated alkyl acids on cellular responses of MCF-10A mammary epithelial cells in monolayers and on acini formation in vitro. Toxicol Lett. 2016;259:95-107.

12. Corsini E, Luebke RW, Germolec DR, DeWitt JC. Perfluorinated compounds: emerging POPs with potential immunotoxicity. Toxicol Lett. 2014;230(2):263-70.

13. DeWitt JC, Peden-Adams MM, Keller JM, Germolec DR. Immunotoxicity of perfluorinated compounds: recent developments. Toxicol Pathol. 2012:40(2):300-11.

14. Dong GH, Tung KY, Tsai CH, Liu MM, Wang D, Liu W, Jin YH, Hsieh WS, Lee YL, Chen PC. Serum polyfluoroalkyl concentrations, asthma outcomes, and immunological markers in a case-control study of Taiwanese children. Environ Health Perspect. 2013;121(4):507-13.

15. Humblet O, Diaz-Ramirez LG, Balmes JR, Pinney SM, Hiatt RA. Perfluoroalkyl chemicals and asthma among children 12-19 years of age: NHANES (19992008). Environ Health Perspect. 2014;122(10):1129-33.

16. Weidinger S, Novak N. Atopic dermatitis. Lancet. 2016;387(10023):1109-22.

17. Barnetson RSC, Rogers M. Childhood atopic eczema. BMJ. 2002;324(7350):1376-9.

18. Sebõk B, Schneider I, Primary Care Paediatricians in Baranya C, Harangi F. Familiar and environmental factors influencing atopic dermatitis in the childhood. J Eur Acad Dermatol. 2006;20(4):418-22.

19. Deckers IA, McLean S, Linssen S, Mommers M, van Schayck CP, Sheikh A. Investigating international time trends in the incidence and prevalence of atopic eczema 1990-2010: a systematic review of epidemiological studies. PLoS One. 2012;7(7):e39803.

20. Narala S, Hata TR. Adult atopic dermatitis with Comorbid atopic disease is associated with increased risk of infections: a population-based crosssectional study. Dermatol Ther. 2017:7(1):111-21.

21. Guo Y, Li P, Tang J, Han X, Zou X, Xu G, Xu Z, Wei F, Liu Q, Wang M, et al. Prevalence of atopic dermatitis in Chinese children aged 1-7 ys. Sci Rep. 2016;6:29751. 
22. Bruckner JV. Differences in sensitivity of children and adults to chemical toxicity: the NAS panel report. Regul Toxicol Pharmacol. 2000;31(3):280-5.

23. Chan HH, Pei A, Van Krevel C, Wong GWK, Lai CKW. Validation of the Chinese translated version of ISAAC core questions for atopic eczema. Clin Exp Allergy. 2001;31(6):903-7.

24. Wang B, Chen Q, Shen L, Zhao S, Pang W, Zhang J. Perfluoroalkyl and polyfluoroalkyl substances in cord blood of newborns in shanghai, China: implications for risk assessment. Environ Int. 2016;97:7-14.

25. Wang IJ, Hsieh WS, Chen CY, Fletcher T, Lien GW, Chiang HL, Chiang CF, Wu TN, Chen PC. The effect of prenatal perfluorinated chemicals exposures on pediatric atopy. Environ Res. 2011;111(6):785-91.

26. Okada E, Sasaki S, Saijo Y, Washino N, Miyashita C, Kobayashi S, Konishi K, Ito YM, Ito R, Nakata A, et al. Prenatal exposure to perfluorinated chemicals and relationship with allergies and infectious diseases in infants. Environ Res. 2012;112:118-25.

27. Granum B, Haug LS, Namork E, Stolevik SB, Thomsen C, Aaberge IS, van Loveren H, Lovik M, Nygaard UC. Pre-natal exposure to perfluoroalkyl substances may be associated with altered vaccine antibody levels and immune-related health outcomes in early childhood. J Immunotoxicol. 2013;10(4):373-9.

28. Smit LA, Lenters $\mathrm{V}$, Hoyer BB, Lindh $\mathrm{CH}$, Pedersen HS, Liermontova I, Jonsson BA, Piersma AH, Bonde JP, Toft G, et al. Prenatal exposure to environmental chemical contaminants and asthma and eczema in schoolage children. Allergy. 2015;70(6):653-60.

29. Okada E, Sasaki S, Kashino I, Matsuura H, Miyashita C, Kobayashi S, Itoh K, Ikeno T, Tamakoshi A, Kishi R. Prenatal exposure to perfluoroalkyl acids and allergic diseases in early childhood. Environ Int. 2014;65:127-34.

30. Goudarzi H, Miyashita C, Okada E, Kashino I, Chen CJ, Ito S, Araki A, Kobayashi S, Matsuura H, Kishi R. Prenatal exposure to perfluoroalkyl acids and prevalence of infectious diseases up to 4years of age. Environ Int. 2017;104:132-8.

31. Dong G-H, Liu M-M, Wang D, Zheng L, Liang Z-F, Jin Y-H. Sub-chronic effect of perfluorooctanesulfonate (PFOS) on the balance of type 1 and type 2 cytokine in adult C57BL6 mice. Arch Toxicol. 2011;85(10):1235-44.

32. Midgett K, Peden-Adams MM, Gilkeson GS, Kamen DL. In vitro evaluation of the effects of perfluorooctanesulfonic acid (PFOS) and perfluorooctanoic acid (PFOA) on IL-2 production in human T-cells. J Appl Toxicol. 2015;35(5):459-65.

33. Corsini E, Sangiovanni E, Avogadro A, Galbiati V, Viviani B, Marinovich M, Gall $\mathrm{CL}$, Dell'Agli M, Germolec DR. In vitro characterization of the immunotoxic potential of several perfluorinated compounds (PFCs). Toxicol Appl Pharmacol. 2012;258(2):248-55

34. Wielsøe M, Long M, Ghisari M, Bonefeld-Jørgensen EC. Perfluoroalkylated substances (PFAS) affect oxidative stress biomarkers in vitro. Chemosphere. 2015;129:239-45.

35. Rockwell CE, Turley AE, Cheng X, Fields PE, Klaassen CD. Persistent alterations in immune cell populations and function from a single dose of perfluorononanoic acid (PFNA) in C57BI/6 mice. Food Chem Toxicol. 2017;100:24-33.

36. Takacs ML, Abbott BD. Activation of mouse and human peroxisome proliferatoractivated receptors (alpha, beta/delta, gamma) by perfluorooctanoic acid and perfluorooctane sulfonate. Toxicol Sci. 2007;95(1):108-17.

37. Fairley KJ, Purdy R, Kearns S, Anderson SE, Meade B. Exposure to the immunosuppressant, perfluorooctanoic acid, enhances the murine $\lg E$ and airway hyperreactivity response to ovalbumin. Toxicol Sci. 2007;97(2):375-83.

38. DeWitt JC, Shnyra A, Badr MZ, Loveless SE, Hoban D, Frame SR, Cunard R, Anderson SE, Jean Meade B, Peden-Adams MM, Luebke RW, Luster MI. Immunotoxicity of Perfluorooctanoic acid and Perfluorooctane Sulfonate and the role of Peroxisome Proliferator-activated receptor alpha. Crit Rev Toxicol. 2009;39(1):76-94.

39. Goudarzi H, Araki A, Itoh S, Sasaki S, Miyashita C, Mitsui T, Nakazawa H, Nonomura K, Kishi R. The Association of Prenatal Exposure to Perfluorinated chemicals with Glucocorticoid and androgenic hormones in cord blood samples: the Hokkaido study. Environ Health Perspect. 2017;125(1):111-8

40. Kapoor A, Petropoulos S, Matthews SG. Fetal programming of hypothalamicpituitary-adrenal (HPA) axis function and behavior by synthetic glucocorticoids. Brain Res Rev. 2008;57(2):586-95

41. Waffarn F, Davis EP. Effects of antenatal corticosteroids on the hypothalamicpituitary-adrenocortical axis of the fetus and newborn: experimental findings and clinical considerations. Am J Obstet Gynecol. 2012;207(6):446-54.

42. Da Silva JAP. Sex hormones and Glucocorticoids: interactions with the immune system. Ann N Y Acad Sci. 1999;876(1):102-18.

43. Yang Zhou L-WH, Zhengmin (Min) Q, Chang J-J, King C, Paul G, ShaoLin P-CC, Lee $\mathrm{YL}$, Dong $\mathrm{G}-\mathrm{H}$. Association of perfluoroalkyl substances exposure with reproductive hormone levels in adolescents: by sex status. Environ Int. 2016;94:7.
44. Zhong SQ, Chen ZX, Kong ML, Xie YQ, Zhou Y, Qin XD, Paul G, Zeng XW, Dong $\mathrm{GH}$. Testosterone-mediated endocrine function and $\mathrm{TH} 1 / \mathrm{TH} 2$ cytokine balance after prenatal exposure to Perfluorooctane Sulfonate: by sex status. Int J Mol Sci. 2016;17(9):1509.

45. Zhou Y, Hu L-W, Qian Z, Geiger SD, Parrish KL, Dharmage SC, Campbell B, Roponen M, Jalava P, Hirvonen M-R, et al. Interaction effects of polyfluoroalkyl substances and sex steroid hormones on asthma among children. Sci Rep. 2017;7(1):899.

46. Mak YLTS, Yeung LW, Lu G, Jin L, Yang Y, Lam PK, Kannan K, Yamashita N. Perfluorinated compounds in tap water from China and several other countries. Environ Sci Technol. 2009:43(13):4824-289.

47. Jun Zhang LY, Meikle S, Zheng J, Sun W, Li Z. Cesarean delivery on maternal request in southeast China. Obstet Gynecol. 2008;111(5):6.

48. Papathoma E, Triga M, Fouzas S, Dimitriou G. Cesarean section delivery and development of food allergy and atopic dermatitis in early childhood. Pediatr Allergy Immunol. 2016;27(4):419-24.

49. Yu M, Han K, Kim DH, Nam GE. Atopic dermatitis is associated with caesarean sections in Korean adolescents, but asthma is not. Acta Paediatr. 2015;104(12):1253-8.

\section{Submit your next manuscript to BioMed Central and we will help you at every step:}

- We accept pre-submission inquiries

- Our selector tool helps you to find the most relevant journal

- We provide round the clock customer support

- Convenient online submission

- Thorough peer review

- Inclusion in PubMed and all major indexing services

- Maximum visibility for your research

Submit your manuscript at www.biomedcentral.com/submit
Biomed Central 